\title{
Investigating the Potential of a Dashboard for Monitoring U.S. Federal Website Accessibility
}

\author{
Jonathan Lazar ${ }^{1}$, Victoria Williams ${ }^{1}$, Jon Gunderson ${ }^{2}$, Tom Foltz ${ }^{2}$ \\ ${ }^{1}$ Dept of Comp. and Information Sciences, Towson University, ${ }^{2}$ Div. of Disability Resources and Education Services, UIUC \\ jlazar@towson.edu, vwilli13@students.towson.edu, jongund@illinois.edu,tmfoltz@gmail.com
}

\begin{abstract}
The U.S. federal government continues to struggle with improving web accessibility for people with disabilities, despite the fact that Section 508 of the Rehabilitation Act has required accessible websites for the federal government since 2001. Researchers have struggled to understand Section 508 due to the lack of transparency in implementation of the law. This article provides two contributions: 1) interviews with five individuals who are Section 508 coordinators in the federal government offer understanding of the potential barriers to dashboard adoption, and 2) data collected on 629 federal websites involving more than 28,000 web pages demonstrate the type of data that potentially could be collected and analyzed in a dashboard. Consistent use of automated tools to create a dashboard of federal web accessibility potentially could bring more attention to the topic and improve accessibility compliance.
\end{abstract}

\section{Introduction}

An emerging topic in the area of e-government, for both researchers and practitioners, is the topic of ensuring that government-developed technologies, including web sites, are accessible for people with disabilities. Governments around the world have struggled with making their web sites accessible for people with disabilities $[1,5,7,9]$. In the United States, Section 508 of the Rehabilitation Act was signed into law in August 1998, with full implementation in 2001. The law was enacted to ensure the removal of barriers to information technology that people with disabilities face on an everyday basis. The main purpose of the law (often known simply as "Section 508") was to ensure all people, including people with disabilities, have equal access to technology. This applies to technologies being used within the government by federal employees, as well as those web sites that form the core of e-government services to citizens.

Section 508 requires that all federal government websites be accessible for people with disabilities. Although this specific law has been in effect since 2001, many federal websites have been determined to be inaccessible, and the effectiveness of the law has been questionable $[5,6,7,9]$. European governments have also had questionable effectiveness in their own e-government accessibility initiatives [1]. Nearly all laws around the world, which require access to egovernment web sites for people with disabilities, reference the international technical standard known as the Web Content Accessibility Guidelines (WCAG).

One of the reasons Section 508 has not been effective is that few data have been collected about compliance, and the data collected by the federal government have not been made public. Openness and transparency could assist policymakers in better understanding of overall compliance with Section 508 and could bring more awareness of the topic to the general public. Because of the lack of openness and transparency, researchers have also struggled to fully understand why implementation of Section 508 (and similar laws) has been so unsuccessful.

In this article, we propose a potential solution for increasing data about web accessibility compliance. Specifically, we propose a government-wide dashboard for web accessibility compliance. After providing background information about U.S. federal website accessibility and dashboards, we provide two empirical contributions. The first contribution is the result of interviews with five individuals who are Section 508 coordinators in the federal government offer understanding of the potential barriers to dashboard adoption. Based on the results of the interviews, one of the potential barriers discussed by the interviewees, producing appropriate data from automated tools to feed into a dashboard, was investigated in further detail. Based on interviewee concerns about the quality of data coming from a dashboard based on automated testing results, a new approach for measurement was attempted, with data collected on accessibility barriers in 629 federal websites involving more than 28,000 web pages, demonstrating the type of data that potentially could be accurately collected and analyzed in a dashboard.

These two separate contributions are unique and differ from previous research on the topic of web accessibility and e-government (such as [5,9,15), which have focused on ascertaining web accessibility compliance at a point in time, without understanding the organizational factors behind the scenes. First, no 
previous publications have ever collected data directly from those responsible for managing compliance within the federal government (the Section 508 coordinators). Second of all, driven by the comments from the interviewees, a new approach to assess compliance with Section 508, using automated tools, was attempted. Rather than determining if all technical standards of Section 508 are met, which requires multiple manual checks and is often an inaccurate or a misleading statistic, this paper takes a different measurement approach, of measuring the presence of accessibility features, which can be considered to partially represent the effectiveness of organizational policies. More details on the methodologies are provided later in the paper.

\section{Background Literature}

\subsection{U.S. federal web accessibility}

Accessibility of e-government web sites is talked about constantly but rarely enforced $[10,15]$. Within the United States, an accessible website is defined as one that strictly follows technical guidelines, such as those in Section 508 (which are derived from the Web Content Accessibility Guidelines). As stated, Section 508 requires that all federal agencies "develop, procure, maintain, or use electronic and information technology" that is fully accessible to those with disabilities. This applies but is not limited to websites, operating systems, hardware, and telecommunications devices. Section 508 regulations are being updated, with the regulation process being managed by the U.S. Access Board. In the newest draft of the regulations (known as the "508 refresh" or "ICT refresh"), the technical standards are directly referenced to be the Web Content Accessibility Guidelines (WCAG) version 2.0. In the newest draft, user performance requirements (i.e., is there an equivalent access method for a class of user?) are given a higher priority than are technical standards. The most recent action on the 508 refresh was the Proposed Rule, which was issued February 18, 2015, with a public comment period ending May 28, 2015 [11].

By law, the U.S. Department of Justice (DOJ) is required to perform a data collection effort every 2 years related to documenting Section 508 compliance across the federal government. From 2004 to 2010, the DOJ did not perform the data collection. In the absence of government data collection, researchers have filled the gap, reporting low levels of web accessibility compliance on federal websites [5,9]. The DOJ issued a report on accessibility in the fall of 2012 [12] that discussed plans and perceptions of compliance but provided no empirical data that assessed the accessibility of federal web pages. So, the data collected by the U.S. Department of Justice does not reflect actual web site accessibility, only plans and perceptions related to accessibility.

Open government is a doctrine that all citizens should have the right to access all documents pertaining to the government. With the issuance of the Open Government Directive in December 2009, the White House and Office of Management and Budget (OMB) required specific steps with which agencies could achieve transparency, participation and collaboration. With this policy in place, government agencies are expected to publish information online and maintain and update such information in a timely manner. However, data on accessibility and Section 508 compliance have never been included in the open government initiatives. In fact, data on specific agency compliance with Section 508 came to the public only because of a series of Freedom of Information Act requests submitted by disability advocates in 20112012 [7]. One of the major findings was that different agencies use a variety of automated accessibility testing tools (at least 20 were mentioned), with no communication between agencies and no testing to determine the validity of the tools or related findings [7]. Many agencies perform no compliance monitoring activities and have no idea whether their websites are compliant with Section 508. Some agencies noted that they investigate only reactively when individual complaints about accessibility are made [7]. In 2013, the White House issued a memo with a plan for improving Section 508 compliance [14]. As a part of improving compliance, agencies are expected to submit basic data about Section 508 compliance every 6 months to the OMB, but the data are not made public, and there are concerns about the validity of the data submitted (discussed later in this article). Transparency in Section 508 compliance continues to be a challenge, and because of the lack of transparency, researchers also find it challenging to understand why Section 508 implementation has been so unsuccessful. While there was much literature about Section 508 in the late 2000 s, very little has been published about Section 508 since 2011, partially because there has been silence from the U.S. Federal Government, rather than transparency, so very little data has been available to analyze. Recent research from [4] shows that government web sites in the UK and US have made improvements since 1999 (around the time when the first Section 508 regulations were released), but many accessibility violations still exist.

\subsection{Dashboards}

A dashboard is a collection of widgets that gives a quick and clear high-level overview of reports, often using performance indicators. Multiple data indicators are consolidated onto a single screen, allowing decision makers to get a quick overview of 
performance indicators. Dashboards typically integrate data from various sources and organize and display the data in a meaningful way [3]. In most cases a dashboard is displayed on a web page based on data that are directly linked to a database, which allows for real-time updates. Although dashboards can be challenging to create and monitor, their benefits are ones that can greatly benefit government agencies and the public. Dashboards for the U.S. Federal government generally allow users to get an overview of a certain data point across agencies, and then drill down to specific data in specific agencies. Figure 1, gives an example of a dashboard currently in use from the federal government, examining IT infrastructure spending across agencies (from www.ITdashboard.gov).

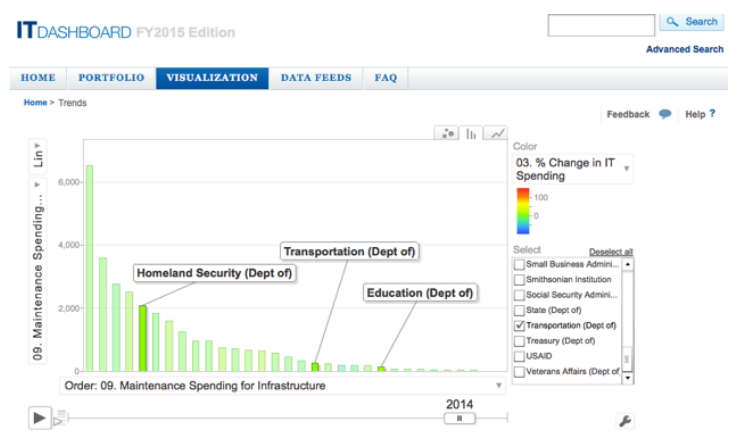

Figure 1. A dashboard examining IT infrastructure spending across U.S. Federal agencies (from www.ITdashboard.gov).

Transparency and dashboards have had some success for government web accessibility compliance in other countries. For instance, in Italy, an automated tool, called the Accessibility Monitoring Application, was developed as a partnership between the University of Bologna and the Emilia-Romagna regional government. The tool regularly collects data on compliance with the Stanca Act for 376 government websites, with reports being available, via a dashboard, to those in charge of website management but not the public [8]. Ganapati states that there are four lessons involved when dealing with government dashboards: 1) data quality is pertinent to the dashboard's credibility, 2) use the best resources when designing the dashboard, 3) performance measures should reflect the goals of the organization, and 4) the effectiveness of a dashboard is solely dependent on its use [3]. Government dashboards have multiple purposes: not only are they used by decision makers inside of government, but they also have a purpose in informing the public about government performance.

\subsection{Automated tools for evaluating web accessibility}

Typically, there are three different approaches to evaluating web accessibility: 1) user testing with assistive technology, 2) expert reviews using assistive technology and 3) accessibility API inspection tools, and automated accessibility tools that inspect the HTML code and/or Document Object Model (DOM) for accessibility features [7]. User testing with assistive technology provides information on both the accessibility and usability of a web resource from a functional perspective (i.e., can information be accessed and/or a task completed independently?). While user testing is ideal, there are 3 limitations: 1 ) it is achievable only when the number of web pages is limited, 2) pages with complete sections that are inaccessible often are missed entirely by users of screen readers if they are not aware of the feature, leaving the appearance that the page/website is accessible, and 3) people with disabilities can generally only identify barriers that relate to their individual disabilities, but not other disabilities. Expert reviews can identify features of the page that are completely inaccessible, but reviewers still face the same time-consuming and resource-intensive process of identifying the accessibility features and issues with a web resource. Automated tools can verify only some of the accessibility requirements found in Section 508 and WCAG 2.0 requirements automatically. For example, does an image have alternative text for people who cannot see (known as an ALT attribute)? Does a form control have a defined label? Is the main content on a web page identified (e.g., H1 element, MAIN landmark, or MAIN element)? Automated tools can act as a filter of accessibility requirements to identify only the manual checks needed for the features found on a page, reducing the number and types of manual checks needed to verify the accessibility of a web resource. (e.g. an automated tool could determine that there is no video on the page, so no human needs to check for captioning or audio description). Automated tools are not good at measuring the quality of ALT text, labels for form controls, or the overall organization of a web resource. Thus, each approach has advantages and disadvantages. Although automated tools have limitations in identifying functional accessibility, they do scale well for testing large numbers of pages for accessibility features, and a recent meta-analysis of research studies documents that, in fact, automated tools are the most common data collection approach used for multi-site evaluation [2]. Automated tools are good to use in a first pass to ensure basic accessibility features are available in a web resource. Once such features are in place, the more time-consuming techniques of user testing and expert reviews can verify functional accessibility and 
usability. Because dashboards that focus on accessibility must have large-scale data collection, automated accessibility testing tools will need to play a large role in any dashboards developed for monitoring accessibility in the U.S. government. Automated tools exist to evaluate websites for accessibility, and federal dashboards exist for other policy-related topics, but automated accessibility testing tools have not been used to create a public dashboard on compliance with Section 508 of the Rehabilitation Act. Because data on web accessibility has never been collected on a large scale in the U.S. federal government and any existing data generally has not been publicly shared, it is important to understand how people involved with Section 508 compliance would react to such a dashboard, what barriers they perceive would exist, and what factors could influence the success of a dashboard for government web accessibility.

\section{Interviews with Section 508 coordinators}

\subsection{Interview methodology}

Each agency within the U.S. federal government has a position known as a Section 508 coordinator. This coordinator has responsibility for the successful implementation of Section 508, ensuring that both public-facing and internal information technology for employees are accessible for people with disabilities. This job responsibility involves everything from managing accessibility requirements in procurement to supervising testing of websites and creating agencywide policies. For some individuals, being the Section 508 coordinator is their primary responsibility, with existing resources, laboratories, and high visibility within an agency. For others, the title is tacked onto their existing job responsibilities, with no resources, and limited availability to have an impact. No previous research has ever collected data directly from those charged with managing accessibility of Federal egovernment web sites: the Section 508 coordinators. To help better understand the potential success factors and barriers involved with implementing a dashboard to monitor U.S. federal web accessibility, interviews with five Section 508 coordinators were done between June 2015 and January 2016. The Section 508 coordinators were recruited through existing contacts of the researchers. While this is not a random or representative sample by any means, this is not a community of professionals who have previously been researched, and due to the often-present threat of lawsuits against federal agencies for non-compliance with disability rights laws, having existing trust between researcher and interviewee is important. On average, the interviews lasted 60 to 75 minutes. Two of the interviews were in person, and three were done via telephone. Anonymity was promised to all participants, so their agencies or departments will not be identified. Because the participants are federal employees in leadership roles, no audio recordings were made; interviewers made only written notes, so statistical analysis of the wording in interviews, even word counts, would not be possible. An interview script was used as a starting point for discussion; questions were asked about the benefits and drawbacks of a dashboard, potential political and financial barriers, and implementation and use of a dashboard. These questions were asked because, while the technology has existed for a number of years, to turn data provided by automated tools into dashboards, it is not known why such an approach has not been taken. These questions were motivated by informal discussions with policymakers before this research took place. From the interviews, a number of common themes were apparent, and the general themes of the interviews are discussed in the next section.

\subsection{Interview Results}

3.2.1 Consistency across the federal government. Nearly all of the participants highlighted the need for increased consistency in how Section 508 is implemented across federal agencies and how automated accessibility testing tools are used as a part of Section 508 enforcement. For instance, two participants highlighted the approaches for web accessibility evaluation at their respective agencies: one noted that automated testing primarily was used, whereas the other, in a different agency, said the agency relies primarily on manual inspection using screen readers. A participant noted, "There is no consistency in how 508 standards are applied, since every agency manages the process differently." It was unclear to participants how dashboards could be used until there was standard data collection throughout the federal government and consistency in the automated testing tools used. One participant noted that "the same tools need to be used throughout the federal government, not different tools." Another participant said, "We need one tool, one dashboard, so that agencies can be fairly compared." A participant noted that "dashboards won't be useful until there are standard approaches for 508 [data collection] across the government." There were many additional comments with the same theme.

The inconsistent use of automated tools was highlighted as a major barrier to the use of dashboards and, more broadly, the success of Section 508 in general. "The technology already exists. The major stumbling block is that we need only one tool for use throughout the federal government."

A participant stated that "automated tools need to be procured government-wide, not at the agency level." It was noted that agencies often do not share information with each other and do not share the best 
practices they have learned about Section 508; the participant noted that "if it's done right, we should replicate it [in other agencies]." For instance, two participants noted the rollout of the Trusted Tester program, which was created by the Department of Homeland Security to train and certify government employees to provide accurate assessments of website accessibility, throughout many agencies in the federal government, and one of those participants stated that "Trusted Tester could level the playing field.". One participant noted that consistency itself could lead to a broader acceptance of the concept of public dashboards: "If there is consistency and the same tools are used throughout government, there might be less hesitation to implementing a public dashboard." Another participant noted that this could be within the general trend of more federal agencies that are measuring progress and publicly posting their progress.

3.2.2 Biannual Reports to the Office of Management and Budget (OMB). Since 2014, all federal agencies have been required to send data twice a year to the OMB on the agencies' Section 508 compliance. Participants had a number of opinions as to whether these data, already being collected, could form the foundation of data in a federal governmentwide dashboard. Although participants generally thought the data could be helpful in the creation of dashboards, some expressed concerned about the quality of the data, confidently asserting that, for instance, the quality of the data is low in the earlier reports submitted to OMB; however, one participant said the quality of the data had improved over time. Another participant stated that in the early stages, the reports actually measured whether or not an agency had a 508 program in place. Multiple participants noted that submitting the report is required, but individuals said "the content is arbitrary," "there is less focus on getting it right and more focus on getting it done," and "the numbers are sometimes 'fudged'." Participants noted it is unclear how OMB uses the data in these reports. For instance, neither the Section 508 coordinators nor the Chief Information Officers (CIOs) receive feedback from OMB: there is no communication about whether the agencies are meeting goals or formatting their data submissions correctly.

3.2.3 A public dashboard vs. a private dashboard. Participants had strong feelings about whether a dashboard should be private, only for internal government use, or instead be available to the public, as many other dashboards are. Two participants noted that some federal agencies have internal dashboards used for management of Section 508 compliance, but the data are not publicly available. Some of the participants said that agencies would be reluctant to share internal data publicly. Participants noted that CIOs might support the development of dashboards for private but perhaps not public use because they would not want a low accessibility score from their agency to be known to the public. Multiple participants wondered if the fear of lawsuits would deter agencies from publicly posting the data because it would mean publicly admitting they were not in compliance with Section 508. As one participant stated, "it's hard to "air our laundry' outside of the government community." Participants noted that having different levels of data available to the public and to policymakers might lead to more support for the concept of public dashboards. However, there was also hesitation on the use of only nonpublic dashboards. One participant noted that "a public dashboard would bring more attention to [Section] 508," but another said that "if only [Section] 508 coordinators see the dashboard data, it won't make any difference. CIOs need to see it." One participant said some agencies might be more likely to be open in their data reporting if they employ a high number of people with disabilities.

3.2.4 Who should coordinate? As mentioned in previous research articles, the responsibility for Section 508 is distributed throughout the federal government (including, for various portions, the White House, the OMB, the U.S. Access Board, and the U.S. DOJ) [3]. Because of the distributed nature of Section 508 responsibility, it is unclear which agency would coordinate a potential dashboard for accessibility. Also unclear is how a standard automated testing tool for use throughout government would be selected. Some participants said OMB was the right agency to coordinate, commenting that "OMB is a really powerful force, and the only one that could take responsibility for a dashboard," "OMB controls the budget, so there's lots of power and clout," and "You would need cooperation from OMB." Other participants said "DOJ would be the ideal coordinator because of the respect that they have [throughout government]. But DOJ would need to be involved with data collection, analysis, and enforcement." The participant probably is alluding to the DOJ having responsibility for data collection but no enforcement authority (unlike the Americans with Disabilities Act, which also has coverage of web sites, for which the DOJ does have enforcement power). Multiple participants also suggested the U.S. Access Board should be involved because of the Board's technical expertise and perceived trust. Some participants suggested that the agencies with expertise in accessibility and Section 508 compliance but no statutory involvement in government-wide compliance, such as Department of Homeland Security, Social Security Administration, Veterans Administration, National Science Foundation, and Department of Education [all suggested by participants], should be given leadership roles; however, multiple participants noted that General Services Administration (GSA) 
should not be given responsibility for a dashboard or, more generally, Section 508 compliance. Despite the negative view of the capabilities of GSA, one participant noted, "If GSA buys into the dashboard, other agencies would follow." It was unclear where funding for the development of a dashboard would come from, although participants said that funding would be required to make a government-wide dashboard a reality. Finally, one participant suggested that it would be good for OMB to find an external company to develop and run the dashboard.

3.2.5 About Section 508 coordinators. Participants were asked about the potential role of Section 508 coordinators in the implementation of dashboards but instead gave feedback about the nature of Section 508 coordinators. For instance, it was noted that Section 508 coordinators are friendly and help each other; they used to hold annual face-to-face meetings, but those meetings have not occurred recently. However, a major weakness of Section 508 is that a lot of the coordinators have the responsibility added to their fulltime jobs, so they do not have sufficient time to dedicate to their Section 508 responsibilities. It was suggested that all Section 508 coordinators should have at least half to full time dedicated to their Section 508 coordination responsibilities. Participants stated more generally that when an individual is assigned the responsibility for Section 508, it is important to ensure he or she has the training needed. One participant stated, "There are many 508 coordinators but few 508 programs." Another participant commented that "many 508 coordinators are not trained in program management." Finally, one participant commented that "508 coordinators are often left out of the loop and only brought in at the last minute."

3.2.6 Accuracy of automated tools. Because automated tools potentially would form the foundation of data collection for a dashboard, participants had many comments about the accuracy of automated tools. For instance, one participant noted that "automated tools only find $20 \%-25 \%$ of the errors," and another said, "There are false positives; there are no perfect tools, [and you need to] be open to that."

There were many comments relating to a dashboard being only as good as the accuracy of the automated tools used for data collection. For instance, one participant stated, "Agencies using automated tools have tons of data but are uncertain about the correctness [of that data]," and another said, "Emphasis needs to be put on making sure that reports are accurate." A participant stated, "A dashboard could help only if testing was reliable, accurate, and consistent." Concern also was expressed about the ramifications of a dashboard using data of questionable validity. One participant said that "false positives would not be grasped well," and another stated that "once data from an automated tool becomes discredited, the whole project could be lost." It was noted that dashboards, in the past, were not wellaccepted because of false-positive results, and "it was detrimental to the success of the dashboard." In that theme, one participant said it would be important to "address the validity of the data and the perception [of the validity]."

Multiple participants said automated tools would not, by themselves, be sufficient; some form of human validation would be needed to determine "if the automated tools have accurate results." For instance, one participant said that "a dashboard alone could not work, [there needs to be] a means for verification." Two participants noted that any type of human validation should be selected randomly, and one participant suggested that at least half of all federal web pages should be validated by humans. Final suggestions from participants on the topic of the accuracy of automated tools included the notion that automated tools do not work well with firewalls and that "the quantitative metrics to be used in data collection for the dashboard need to be clear, as will the thresholds for success."

3.2.7 Technical aspects of dashboards. There were surprisingly few comments about technical aspects of the dashboard because participants saw so many other potential barriers to success. Multiple participants noted that a potential dashboard for Section 508 compliance itself would need to be accessible; otherwise, it could "lead to a public relations nightmare." For instance, it was noted that years ago, GSA tried to create a dashboard for accessibility but had no idea what the automated tools were good at, so the effort was not a success (the participant suggested that GSA should have been more open about the strengths and weaknesses of automated tools). The accessibility of the dashboards, aside from being a publicity problem, is important (as multiple participants noted) because many of the Section 508 coordinators have disabilities. Other comments related to technical aspects of the dashboard include the suggestion of monthly scans [of each web page] for accessibility and the use of an easy-to-understand interface - for instance, one that uses red/yellow/green lights, which often are used for dashboards.

3.2.8 Potential impact of a dashboard. All participants said the main reason to use dashboard would be because it could potentially improve Section 508 compliance. One participant commented, "A dashboard, if measured properly, could have an impact." Some comments related to the role of CIO. One participant said, "A CIO would be politically impacted if their agency was reported to be doing poorly." The connection between results of compliance and budget was noted to be an important relationship. As one participant said, "There might be public humiliation, but it could lead to budget implications, 
which would be very important." Another comment was that a dashboard would be helpful only if high level managers use it and were allocated resources for it. One participant noted the potential downside to a dashboard:" When you focus on websites, automated tools and dashboards, CIOs often don't see the nonweb side of Section 508." That participant was concerned that CIOs would focus only on the dashboards and automated tools because they were getting attention due to the dashboard and potentially could ignore the accessibility of software applications, operating systems, hardware, and other office devices.

How would the success of a dashboard be determined? One participant said that positive trend lines in Section 508 compliance after a dashboard was implemented would mean success. Another participant said, "I would measure success of a dashboard if consistent results were $95 \%$ or above compliant [with Section 508]." One participant said a dashboard potentially could influence policymakers, and another postulated that implementation of a dashboard would not affect the rule-making process for Section 508 but could have an impact on the rule-making process for the Americans with Disabilities Act because the topic of web accessibility would receive more attention. Finally, one participant noted that a dashboard might help more people understand the topic of accessibility: "People inherently understand privacy and security but don't understand Section 508 and how it relates to their world."

3.2.9 Receptiveness to a dashboard. Concerns were expressed about hesitation or political pushback around the idea of implementing a dashboard that potentially could highlight failure. For instance, one participant noted that when agencies are not doing well with Section 508, you would expect that they would "push back" about publicly posting their data. Another participant noted that "when more federal agencies have mature 508 programs, there may be less hesitation to publicly post data." The potential negative publicity was noted by a participant who said, "We don't want to see ourselves in the front of the Washington Post." One participant said the hesitation most likely would come from the CIOs, "I think that hesitation to publicly post data would come from CIOs. Most middle-level managers would love the transparency." One participant said, "508 compliance is not always valued as important... a dashboard is the way to drive success with 508." One participant said a dashboard might be helpful because "If you want to get something done, you've got to measure it."

Other suggestions for improving the potential success of a dashboard included being clear about the credibility of the data, planning for buy-in from agencies, and ensuring that the dashboard is free to the agencies (because expenses likely would dampen enthusiasm). Finally, one participant said that having all of the results publicly available on a dashboard would increase competition among all agencies, leading to improved compliance with Section 508

\subsection{Discussion on Interviews}

The participants in the interviews provided a wealth of data about barriers to Section 508 compliance and the potential implementation of a dashboard. Some of the barriers mentioned were political, managerial, or financial and will require future investigation. For the next stage of research, we selected one of these topics to investigate: the topic of data accuracy and validity of data being collected by automated tools. This issue came up multiple times during the interviews and dealt with the clear need for the use of automated tools (because they are the only evaluation method with scalability over tens of thousands of web pages), but concerns were expressed about using the automated tools appropriately and ensuring validity of the data. Interviewees specifically noted that the metrics often used, of whether web pages specifically met all Section 508 requirements, according to the automated tools, was a misleading metric. Furthermore, such a metric isn't helpful to policymakers, who are interested in the big picture (questions such as, are we making progress on accessibility? Which agencies are doing a better job?), rather than technical level concerns about how many accessibility violations are occurring on a given page and need to be fixed, which is more interest to web developers, webmasters, and software engineers. Given that automated testing tools will need to be a part of any realistic plan for monitoring web accessibility in the federal government, what are the types of measures that could be accurately assessed by automated tools? What types of data could be fed into a dashboard? What would some of the metrics look like, and how could they influence decision makers in government?

In the previously published literature, various methods are used to quantify the accessibility of a web page or web site. For instance, Youngblood [15] measures the number of accessibility errors as defined by an automated tool. Hanson and Richards [4] chose the percentage of violations of all sites in their sample. Others have attempted to come up with metrics that are computed, for instance the Web Accessibility Barrier score is a weighted average involving the number of accessibility barriers and barriers are prioritized based on how the violation is ranked within the WCAG, and the Web Accessibility Quantitative Metric prioritizes barriers that can be evaluated by automated tool, over those that require some human checking [13]. Vigo and Brajnik clearly state the challenges: "we are faced with a conundrum: on the one side we have quick reliable but potentially invalid ways to measure 
accessibility, on the other side we have expensive methods to evaluate accessibility, subject to a smaller degree of invalidity and unreliability..." [13, p.2].

In the data collection effort detailed in the next section, we attempt to demonstrate the types of data that could be accurately collected by automated tools to be fed into a government dashboard, which would be of use to policymakers and the general public, measuring web accessibility throughout the federal government.

\section{Data collection using automated tools 4.1 Research methodology}

A list of federal websites was identified from an index of websites on USA.gov, the U.S. government's official web portal. A potential of 1,094 websites were identified; analysis found broken and redundant links, so the total usable URLs was reduced to 629 . The 629 URLs were analyzed using the Functional Accessibility Evaluator (FAE) application to spider the pages of each website in September 2015, resulting in a total of 28,429 webpages analyzed. Three rules were selected from WCAG 2.0 as ones that could be useful in developing a dashboard application to track the accessibility of federal websites, but other rules could be effectively evaluated using automated tools. The subset of rules in this article were selected based on their ability to provide clear pass/fail results. For each selected rule, a score of each website was calculated based on the implementation of the rule. A score of 0 means that the rule was not implemented on any of the pages within a website. A score of 100 means that every page of the website implemented the rule requirements (e.g., all images on all the pages had an ALT attribute). Given that the U.S. Federal Government has yet to formally adopt WCAG 2.0 as the standard for Section 508 (the current technical standard for Section 508 is a modified version of WCAG 1.0) and given that automated tools cannot fully evaluate for compliance with any complete set of guidelines, the results presented here do not identify levels of legal conformance with Section 508. However, these results help illustrate the types of data that could be reliably provided by a dashboard using automated tools for data collection. The approach taken here is different from previously published articles. Previously published research generally tries to ascertain compliance with a set of technical standards using automated tools, saying that a web page is or is not compliant [e.g. 5,10,15]. As the interviews highlighted, trust of these automated tools in determining actual compliance is low, the Section 508 coordinators were generally suspicious of the results, and with the large number of manual checks involved, those interviewed did not have a high level of confidence that the tools could be used to show legal compliance. The metric utilized in the current research is different: the data attempts to ascertain the level of implementation of accessibility features, to understand the effectiveness of Section 508 implementation in a given agency, a goal which is much more appropriate and realistic for the automated tools. These results do not provide a complete picture of accessibility of U.S. federal websites but do provide insight into the current level of implementation of particular accessibility features on a large number of federal webpages.

\subsection{Results of Automated Accessibility Testing}

4.2.1 Rule 1: ALT attribute for Image. If any rule is the poster child of web accessibility, it is the ALT attribute for IMG element. It is easy to understand that people who cannot see an image need some type of text description of the image. It is also easy for automated tools to determine if the ALT attribute has been set on an IMG element, although automated tools cannot identify the quality of the text content in describing the image. An empty ALT attribute (ALT $="$ ") is allowed by Section 508 and WCAG 2.0 to identify an image that is purely decorative. Figure 2 shows that ALT attribute on an IMG element is widely implemented across the federal government, with more than 500 websites showing that it is used at least $80 \%$ of the time.

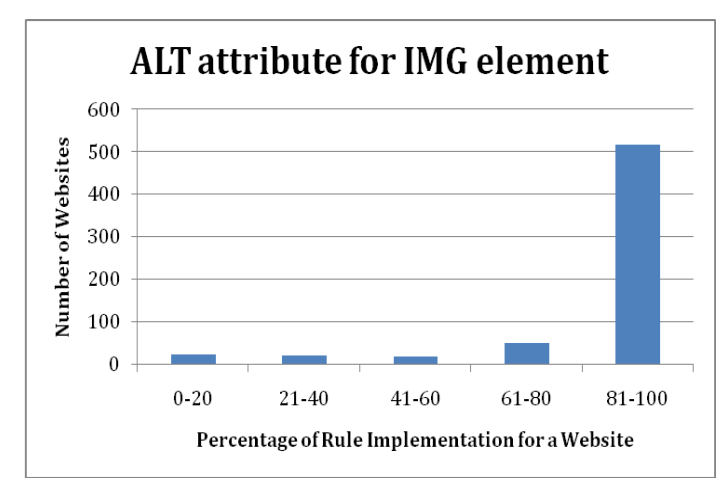

Figure 2. Number of websites listed according to the percentage of IMG elements with an ALT attribute

4.2.2 Rule 2: Labels for Form Controls. Labels for form controls orient users of screen readers to the purpose of a form control input and, if LABEL elements are used, provide a larger clickable area to move keyboard focus to form controls and check/uncheck radio buttons and checkboxes. Unlike ALT text for images, for which an empty ALT text could satisfy the rule, a form control label must have text content to meet Section 508 and WCAG 2.0 accessibility requirements. Figure 3 shows that the 
implementation is bimodal, with 282 websites having labels on at least $80 \%$ of their form controls but 171 websites having less than $20 \%$ of their form controls with labels.

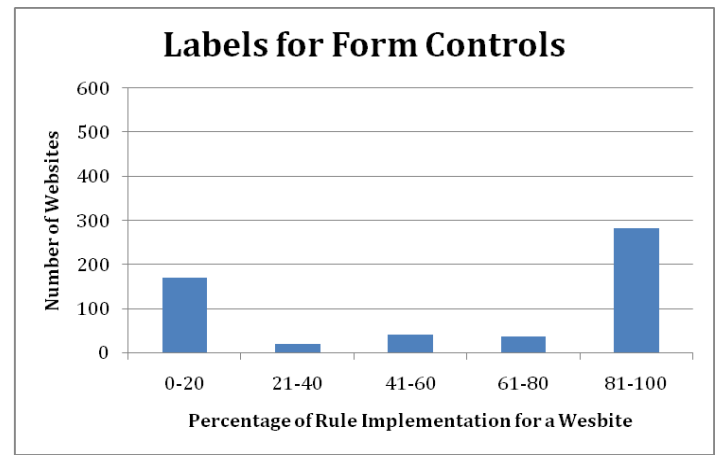

Figure 3. Number of websites listed according to the percentage of form controls with labels

4.2.3 Rule 3: Page has H1 heading. A best practice in using heading elements on a web page is to use the $\mathrm{H} 1$ element to identify the main content of a web page. This allows users of assistive technologies to navigate easily to the start of the main content of a page. This rule looks for the definition of an $\mathrm{H} 1$ element on each page of a website. Figure 4 shows that more than twothirds of federal websites evaluated (366 websites) consistently use an $\mathrm{H} 1$ element and that about onefourth of federal websites (164 websites) do not consistently use $\mathrm{H} 1$ elements.

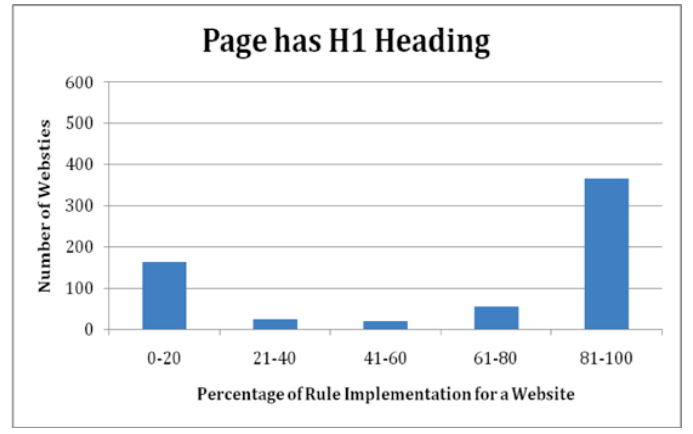

Figure 4. Number of websites listed according to the percentage of pages with an h1 heading

4.2.4 Discussion on automated testing. The data from the three examples shows that distributions generally tended to be bimodal. Many websites had $81 \%$ to $100 \%$ rule implementation, and many had $0 \%$ to $20 \%$ rule implementation, but few were in between. These data relate to the comment from the Section 508 coordinator who said, "There are many 508 coordinators but few 508 programs.” It seems agencies implement these rules throughout their website or do not, but there is little partial implementation. Federal agencies do not seem to be doing it "halfway," meaning that training and awareness by the employees of a federal agency may have a great impact in improving web accessibility.

\section{Summary}

The data collected from automated evaluations demonstrates that, from a technical point of view, useful information related to web accessibility potentially could be collected for the development of a dashboard. An automated tool periodically could sample multiple websites and pages to provide an estimate of the level of implementation of accessibility for portions of the requirements of current Section 508 requirements and the future requirements of WCAG 2.0. The dashboard information would be useful to agencies in helping them develop policies, set priorities, and allocate resources. Additional work is needed to determine how non-automated rules that require manual checking could be used to aid the understanding of the accessibility of an agency's web resources and how that data would need to be implemented into a dashboard.

From a technical point of view, one of the issues with a website accessibility dashboard is the rules used to estimate accessibility compliance. WCAG 2.0 is a standard for the needs of people with disabilities and is technology agnostic. Complying with WCAG 2.0 requirements for a specific technology, such as HTML4, Flash, PDF, or HTML5, requires matching the accessibility features of the technology to the WCAG 2.0 requirements; for some technologies, there may be no way to meet a particular WCAG 2.0 requirement. For instance, what if there are newer technological approaches that meet the functional performance requirements but not the technical standards? Furthermore, accessibility experts often disagree about which techniques are required or merely a best practice for meeting WCAG 2.0 requirements. Much of the disagreement is based on the largely remedial approach to accessibility by which accessibility is addressed late in the development process or after a product has been released. It is similar to building a sidewalk and then retrofitting it with curb cuts. Best practices for web accessibility cost little or nothing if they are built in to the project plan and development process. Another issue regarding rules is that web and assistive technologies are constantly evolving, and rules need to adapt to the latest web accessibility techniques and specification features. The selection of rules for a dashboard is complicated by the large number of accessibility requirements that require manual checking. In general (not just in the e-government realm), people want 
pass/fail rules that can be automated, but these can be applied to only about $30 \%$ to $35 \%$ of WCAG 2.0 requirements (ones which were utilized in the current data collection effort).

The barriers to successful implementation of a dashboard that were investigated, with the use of data collection from an automated tool, relate only to the technical aspects of such an effort. Based on the interviews reported, there also are many potential political, financial, and managerial barriers. How would the development of a dashboard be funded? Given that no agency has enforcement power, strictly speaking, for Section 508, how would such a dashboard be rolled out? How would existing data collection efforts be integrated into the dashboard if there is no consistency in how data are collected? Although there potentially could be many benefits to having a dashboard that monitors web accessibility in the U.S. federal government, much more research is needed to determine how these barriers could be overcome.

\section{References}

[1] Andreasson, K., and Snaprud, M. (2014). The european e-government web accessibility divide. In Proceedings of the 8th International Conference on Theory and Practice of Electronic Governance (ICEGOV '14), 391-394.

[2] Baazeem, I. and Al-Khalifa, H. (2015). Advancements in web accessibility evaluation methods: how far are we? In Proceedings of the 17th International Conference on Information Integration and Web-based Applications \& Services (iiWAS '15), Article 90,5 pages.

[3] Ganapati, S. (2011). Using Dashboards In Government. The IBM Center for the Business of Government (Fostering Transparency and Democracy Series), available at: http://faculty.cbpp.uaa.alaska.edu/afgjp/PADM601\%620Fall $\% 202011 /$ Use $\% 202020$ of $\% 202020$ Dashboards $\% 202020 \mathrm{in} \%$ 202020Government.pdf

[4] Hanson, V. L., \& Richards, J. T. (2013). Progress on website accessibility? ACM Transactions on the Web (TWEB), 7(1), 2.

[5] Jaeger, P. (2006). Assessing Section 508 compliance on federal e-government Web sites: A multi-method, usercentered evaluation of accessibility for persons with disabilities. Government Information Quarterly, 23 (2), 169190.

[6] Jaeger, P. T. and Matteson, M. (2009). e-Government and Technology Acceptance: the Case of the Implementation of Section 508 Guidelines for Websites. Electronic Journal of e-Government 7(1), 87-98.

[7] Lazar, J., Goldstein, D. and Taylor, A. (2015). Ensuring Digital Accessibility Through Process and Policy. Amsterdam: Morgan Kaufmann/Elsevier.

[8] Mirri, S., Muratoir, L. and Salomoni, P. (2011). Monitoring Accessibility: Large Scale Evaluations at a Geo Political Level. Proceedings of the ACM Conference on Accessible Computing (ASSETS), 163-170.

[9] Olalere, A. and Lazar, J. (2011). Accessibility of U.S. federal government home pages: Section 508 compliance and site accessibility statements. Government Information Quarterly, 28(3) 303-309.

[10] Rubaii-Barrett, N., \& Wise, L. R. (2008). Disability access and e-government: An empirical analysis of state practices. Journal of Disability Policy Studies 19(1), 52-64.

[11] U.S. Access Board. (2016). About the ICT Refresh. available at: https://www.access-board.gov/guidelines-andstandards/communications-and-it/about-the-ict-refresh

[12] U.S. Department of Justice. (2012). Section 508 Report to the President and Congress: Accessibility of Federal Electronic and Information Technology. Available at: http://www.ada.gov/508/508_Report.htm

[13] Vigo, M., \& Brajnik, G. (2011). Automatic web accessibility metrics: Where we are and where we can go. Interacting with Computers, 23(2), 137-155.

[14] White House. (2013). Strategic Plan for Improving Management of Section 508 of the Rehabilitation Act Available

at: http://www.whitehouse.gov/sites/default/files/omb/procurem ent/memo/strategic-plan-508-compliance.pdf

[15] Youngblood, N., \& Mackiewicz, J. (2012). A usability analysis of municipal government website home pages in Alabama. Government Information Quarterly, 29(4), 582588 . 